\title{
Formation of a magnetite/hematite epitaxial bilayer generated with low energy ion bombardment
}

Cite as: Appl. Phys. Lett. 110, 093103 (2017); https://doi.org/10.1063/1.4977491

Submitted: 05 August 2016 • Accepted: 14 February 2017 • Published Online: 28 February 2017

S. Ruiz-Gómez, A. Serrano, I. Carabias, et al.
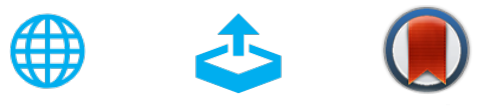

View Online

Export Citation

\section{ARTICLES YOU MAY BE INTERESTED IN}

Reversible oxidation-reduction of epitaxial iron oxide films on $\mathrm{Pt}(111)$ : Magnetite-hematite interconversion

The Journal of Chemical Physics 152, 054701 (2020); https://doi.org/10.1063/1.5136322

Highly $\mathrm{Bi}$-doped $\mathrm{Cu}$ thin films with large spin-mixing conductance

APL Materials 6, 101107 (2018); https://doi.org/10.1063/1.5049944

Room temperature skyrmion ground state stabilized through interlayer exchange coupling

Applied Physics Letters 106, 242404 (2015); https://doi.org/10.1063/1.4922726

Challenge us.

What are your needs for periodic signal detection?

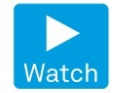

- Zurich

Instruments

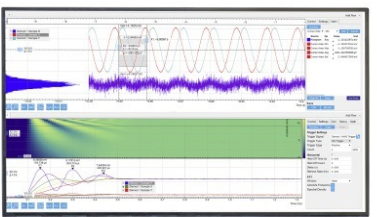

. 


\title{
Formation of a magnetite/hematite epitaxial bilayer generated with low energy ion bombardment
}

\author{
S. Ruiz-Gómez, ${ }^{1,2}$ A. Serrano, ${ }^{3}$ I. Carabias, ${ }^{4}$ M. A. García, ${ }^{3,5}$ A. Hernando, ${ }^{1,5}$ \\ A. Mascaraque, ${ }^{1,2,5}$ L. Pérez, ${ }^{1,2,5}$ M. A. González Barrio, ${ }^{1,2,5}$ \\ and O. Rodríguez de la Fuente ${ }^{1,2,5}$ \\ ${ }^{1}$ Departamento de Física de Materiales, Universidad Complutense de Madrid, 28040 Madrid, Spain \\ ${ }^{2}$ Unidad Asociada IQFR(CSIC)-UCM, 28040 Madrid, Spain \\ ${ }^{3}$ Instituto de Cerámica y Vidrio, CSIC, 28049 Madrid, Spain \\ ${ }^{4}$ C.A.I. de Difracción de Rayos X, Universidad Complutense de Madrid, 28040 Madrid, Spain \\ ${ }^{5}$ Instituto de Magnetismo Aplicado, UCM-CSIC-ADIF, 28230 Las Rozas, Madrid, Spain
}

(Received 5 August 2016; accepted 14 February 2017; published online 28 February 2017)

\begin{abstract}
We have used a low-energy ion bombardment to fabricate an epitaxial single-crystalline magnetite/ hematite bilayer grown on $\mathrm{Au}(111)$. This non-conventional fabrication method involves the transformation of the upper layers of a single-crystalline hematite thin film to single-crystalline magnetite, a process driven by the preferential sputtering of oxygen atoms and favoured by the good structural matching of both phases. We show the reversibility of the transformation between hematite and magnetite, always keeping the epitaxial and single-crystalline character of the films. The magnetic characterization of the bilayer grown using this method shows that the magnetic response is mainly determined by the magnetite thin film, exhibiting a high coercivity. Published by AIP Publishing.

[http://dx.doi.org/10.1063/1.4977491]
\end{abstract}

The production of oxide heterostructures is a prominent topic in the development of new materials. While oxides already display a huge range of functionalities, ${ }^{1}$ their combination in a same material may give rise to novel phenomena not displayed by either of the constituent oxides alone. This can be due to the emergence of new physical processes at the interfaces $^{2}$ or to coupling and/or exchange effects between both materials. ${ }^{3,4}$ Although there are several well-established techniques used to generate oxide layers and interfaces (for instance, Molecular Beam Epitaxy (MBE), sputtering or Pulsed Laser Deposition (PLD)), the continuous improvement of the existing techniques or the development of new synthesis methods is mandatory. An interesting subset of oxide interfaces is that formed by two simple oxides with the same cation but in a different oxidation state. To obtain such system, we present a different approach in this work: the use of Low Energy Ion Bombardment (LEIB). The method has been applied to the $\mathrm{TiO}(001) / \mathrm{TiO}_{2}(110)$ system, ${ }^{5}$ and we show here its application to iron oxides: a $\mathrm{Fe}_{3} \mathrm{O}_{4} / \alpha-\mathrm{Fe}_{2} \mathrm{O}_{3}$ single-crystalline epitaxial bilayer. The presence of a magnetite/hematite interface has been reported, for instance, in a milled powder, ${ }^{6}$ in polycrystalline thin films, ${ }^{7,8}$ in core-shell nanoparticles, ${ }^{9}$ or in hybrid microcubes. ${ }^{10}$ Also, several recent works ${ }^{11-13}$ have shown the conversion of $\alpha-\mathrm{Fe}_{2} \mathrm{O}_{3}$ thin films into $\mathrm{Fe}_{3} \mathrm{O}_{4}$ (even reversibly) or of $\mathrm{Fe}_{3} \mathrm{O}_{4}$ islands into $\gamma-\mathrm{Fe}_{2} \mathrm{O}_{3}$ islands. ${ }^{14}$ But this system has not been synthesized yet as a thin epitaxial bilayer. An attractive circumstance is that it comprises an antiferromagnetic (AFM) and a ferrimagnetic (FiM) oxide.

The films have been grown by reactive-MBE in a UHV system, depositing iron from a Fe rod heated by electron bombardment on a $\mathrm{Au}(111)$ substrate. The iron oxide phases have been characterized with Low Energy Electron Diffraction (LEED), ex-situ X-ray Diffraction and Reflectivity (XRD and XRR), and ex-situ Raman microspectroscopy using a
Confocal Raman Microscope (CRM) from Witec (ALPHA 300RA) with a linearly polarized Nd:YAG laser (532 nm). Magnetization curves at different temperatures were measured with a Vibration Sample Magnetometer (VSM) and with a SQUID magnetometer, both from a Quantum Design. The curves at room temperature (RT) were also measured by the Magneto-Optical Kerr effect (MOKE) in longitudinal configuration (p-polarization) with a homemade device.

The generation of the oxide bilayer involves the initial growth of an epitaxial hematite thin film. After its deposition, $\mathrm{Fe}$ is fully oxidized at $550{ }^{\circ} \mathrm{C}$ under an $\mathrm{O}_{2}$ pressure of $2 \times 10^{-4}$ mbar for $30 \mathrm{~min}$. After the growth, the films show a LEED pattern, as shown in Figure 1(a), with the hexagonal $(\sqrt{3} \times \sqrt{3})$ R30 $0^{\circ}$ symmetry of the new spots corresponding to hematite. The second step of the process involves the transformation of the upper layers of hematite into magnetite, using LEIB with $\mathrm{Ar}^{+}$ions at $1.0 \mathrm{kV}$ for $2 \mathrm{~min}$ at $\mathrm{RT}$. After ion bombardment, a diffuse LEED is observed, already showing the structure of magnetite (Figure 1(b)). New spots appear in the position of a $(2 \times 2)-\mathrm{Fe}_{3} \mathrm{O}_{4}$ structure, and those spots of the $(\sqrt{3} \times \sqrt{3}) \mathrm{R} 30^{\circ}$ hematite reconstruction disappear completely. The surface crystalline structure is completely evident after heating at $550{ }^{\circ} \mathrm{C}$ for $10 \mathrm{~min}$ under UHV conditions: a clear and sharp LEED pattern corresponding to magnetite is observed (Figure 1(c)). To check the reversibility of the transformation induced by LEIB, magnetite is exposed at $550^{\circ} \mathrm{C}$ to an $\mathrm{O}_{2}$ pressure of $2 \times 10^{-4}$ mbar for $30 \mathrm{~min}$. Figure $1(\mathrm{~d})$ shows the LEED pattern after the re-oxidation process: a clear $(\sqrt{3} \times \sqrt{3})$ R30 $0^{\circ}$ LEED pattern corresponding to hematite is again observed. The transformation is thus reversible.

It is interesting to note that for smaller ion doses, a coexistence of both hematite $(\sqrt{3} \times \sqrt{3}) \mathrm{R} 30^{\circ}$ and magnetite $(2 \times 2)$ phases is observed (Figure 1(e)), which indicates that 
(a)

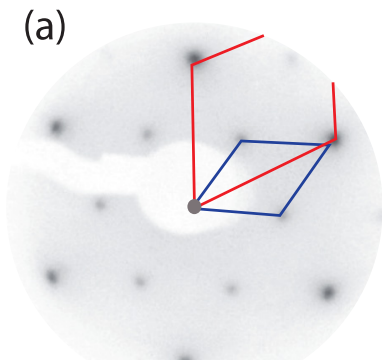

(c)

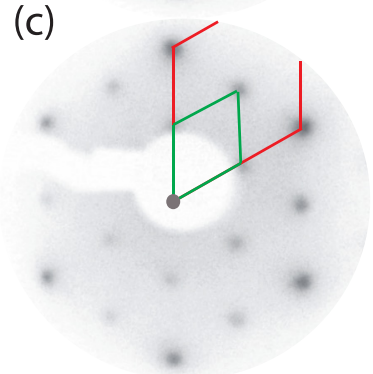

(e)

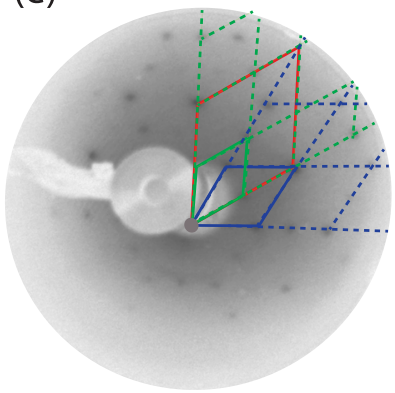

(b)

(d)

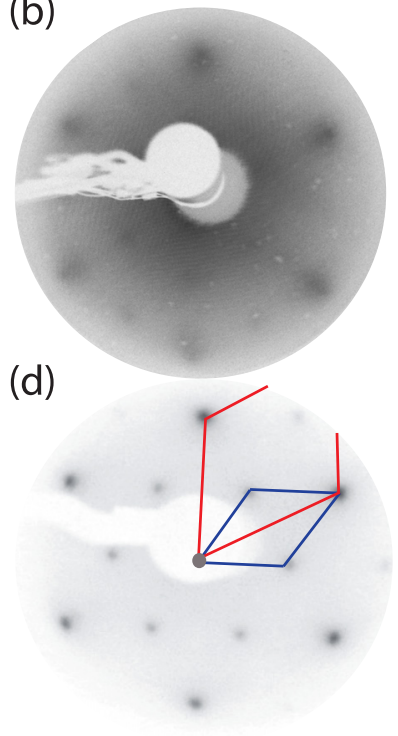

(f)

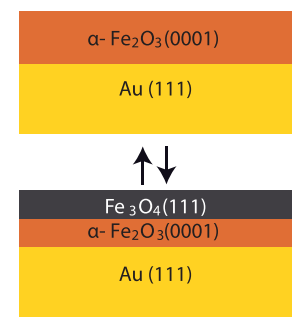

FIG. 1. LEED patterns of (a) the as-grown hematite film, showing the expected $(\sqrt{3} \times \sqrt{3}) \mathrm{R} 30^{\circ}$ symmetry (unit cell in blue) with respect to the $(1 \times 1)$ symmetry of the $\mathrm{Au}(111)$ substrate (in red). (b) Magnetite film after ion bombardment of the hematite thin film in (a). While the spots are broad and diffuse and the background is bright, they already show an emerging new $(2 \times 2)$ symmetry. (c) The magnetite thin film after annealing the sample in (b), with sharper spots and a darker background. (d) Hematite recovered after a re-oxidation process of the magnetite film in (c). (e) Partial transformation of the hematite film, where both $\alpha-\mathrm{Fe}_{2} \mathrm{O}_{3}(0001)$ and $\mathrm{Fe}_{3} \mathrm{O}_{4}(111)$ are shown to co-exist after bombarding the sample for just $1 \mathrm{~min}$. The upper layers of the hematite phase have not been completely transformed. The pattern shows three unit cells: $\mathrm{Au}(111)$ substrate (red), $\alpha-\mathrm{Fe}_{2} \mathrm{O}_{3}(0001)$ (blue), and $\mathrm{Fe}_{3} \mathrm{O}_{4}$ (111) (green). (f) Scheme of the reversible transformation induced: the upper layers of the hematite film are converted into magnetite after ion bombardment, which can be transformed back into hematite by thermal oxidation.

the transformation is gradual and depends on the ion dose. It is also important to remark that the formation of magnetite on the hematite thin film is not the result of a chemical reduction induced by the thermal annealing in UHV carried out after LEIB. The first evidence is that we already observe the diffuse $(2 \times 2)$ pattern of the magnetite before any annealing (Figure 1(b)). The second evidence is that when the same annealing process performed on the hematite sample but without a previous LEIB, the hematite remains unchanged, with no chemical reduction. It is clear that the transformation is induced by the ion bombardment although the crystallinity is improved by thermal annealing.

These results give clear indications of a reversible hematite/magnetite structural conversion of the thin film induced by the ion bombardment and re-oxidation processes assisted with temperature, keeping the epitaxial character of the film.

But this conclusion, based on LEED, is only valid for a shallow depth of the film according to the high surface sensitivity of the technique (around $1 \mathrm{~nm}$ ). Additional techniques are needed to better evaluate the spatial range of the structural transformation.

CRM is a much more local technique but has a much smaller surface sensitivity than LEED. It can thus help us elucidating the depth of the hematite/magnetite conversion especially if we combine it with LEED. For this purpose, we have used two hematite samples with different initial thicknesses: 8 and $14 \mathrm{~nm}$, as measured by XRR. The $8 \mathrm{~nm}$ sample shows both a LEED pattern (not shown here) and a Raman spectrum corresponding to hematite (Figure 2(a), bottom). We can distinguish the seven phonon modes $\left(2 \mathrm{~A}_{1 g}+5 \mathrm{E}_{g}\right)$ allowed in Raman for hematite, ${ }^{15}$ besides other modes reported in the literature. ${ }^{16-18}$ Those include the following: (a) the $\mathrm{LO} \mathrm{E}_{u}$ mode around $671 \mathrm{~cm}^{-1}$ activated by disorder, (b) a magnon excitation at $835 \mathrm{~cm}^{-1}$, and (c) the overtone (2LO $\mathrm{E}_{u}$ mode) around $1332 \mathrm{~cm}^{-1}$, resonantly enhanced and quite characteristic.

After bombardment, the LEED pattern (not shown) displays the expected symmetry of magnetite; all Raman modes of hematite disappear, and a completely new spectrum comes out (Figure 2(a), top). All the new vibrational bands correspond to the five allowed Raman modes $\left(\mathrm{A}_{1 g}+\mathrm{E}_{g}+3 \mathrm{~T}_{2 g}\right)$ of magnetite. ${ }^{15,17,19}$ No bands from hematite are present. These observations clearly account for a full structural transformation of the whole hematite thin film into magnetite.

To investigate a gradual transition from one oxide to the suboxide, we have used the thicker film $(14 \mathrm{~nm})$ and performed shorter consecutive ion bombardments, also combining LEED and CRM in between (Figure 2(b)). Initially, the as-grown sample shows the Raman spectrum of hematite. After the first ion bombardment, while the LEED pattern only displays the symmetry corresponding to magnetite, the Raman spectrum still identifies the hematite modes although we can distinguish new emerging bands around $677 \mathrm{~cm}^{-1}$ $\left(\mathrm{A}_{1 g}\right)$ and $553 \mathrm{~cm}^{-1}\left(\mathrm{~T}_{2 g}\right)$, both originating from magnetite. These observations indicate that while the upper layers have fully transformed into magnetite, hematite persists in the lower layers of the film. This is the first indication of the existence of the bilayer. Increasing the ion dose induces a decrease in the hematite bands and an increase in the Raman signal of the magnetite modes (Figure 2(b)). Finally, the full transformation is achieved, with the Raman spectrum showing only the magnetite vibrational modes. The Raman spectra do not show, in any case, traces from maghemite.

XRD can yield information about the epitaxial character or in-plane orientation of the whole film, an information which is not directly retrieved by either the two previous techniques used (LEED and CRM). Unfortunately, in a conventional $\theta / 2 \theta$ configuration, only reflections corresponding to $\mathrm{Au}(111)$ are detected (see inset Figure 3(c)). The reason is that the reflections corresponding to $\alpha-\mathrm{Fe}_{2} \mathrm{O}_{3}(0001)$ and $\mathrm{Fe}_{3} \mathrm{O}_{4}(111)$ are weak, and for the case of hematite, it falls close to the $\mathrm{Au}(111)$ peak. To confirm the epitaxial character of the bilayer in their whole extent, we have performed azimuthal $(\phi)$ X-ray scans in an asymmetric configuration with a fixed tilt angle $(\chi)$ for each material (see scheme in Figure $3(\mathrm{a}))$. For the case of the $\alpha-\mathrm{Fe}_{2} \mathrm{O}_{3}(0001)$ layer, the tilt angle 
(a)

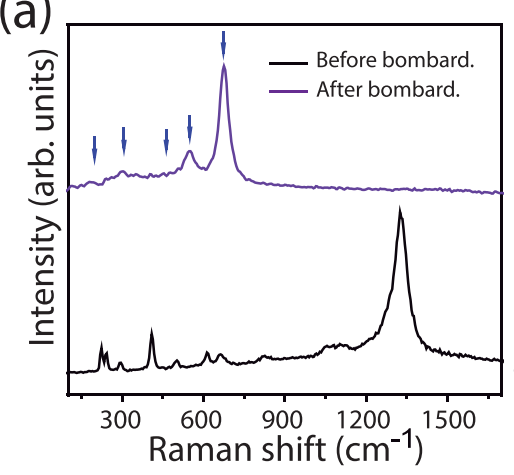

(b)

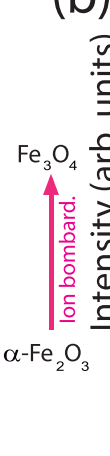

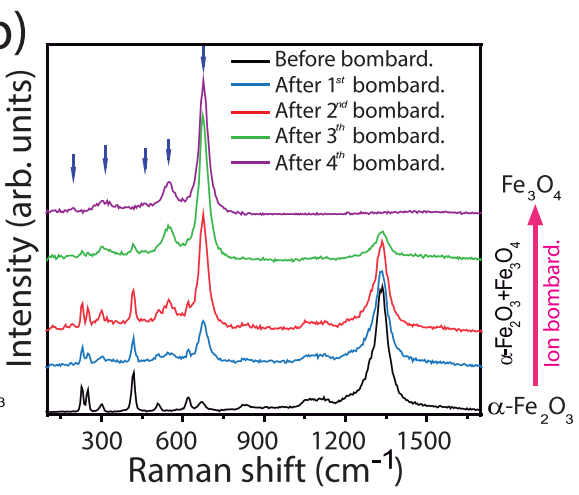

FIG. 2. (a) Raman spectra of the as-grown hematite thin film (black line) and after LEIB (violet line) for an initial thickness of 8 nm. A hematite-to-magnetite full transformation, induced by ion bombardment, is observed. (b) Raman spectra of as-grown hematite (black line) and after sequential ion bombardments with increasing doses (upper lines) for a thicker film with an initial thickness of $14 \mathrm{~nm}$. A hematite-to-magnetite gradual transformation is observed as revealed by the emergence of the Raman modes of magnetite co-existing with those of hematite up to the complete conversion. Blue arrows mark the observed Raman modes of magnetite.

$\chi=38.68^{\circ}$ and, in this configuration, a $\theta / 2 \theta$ scan is carried out to detect the (104) reflection at $2 \theta=33.2^{\circ}$. At this point, a polar $(\phi)$ scan is done. In the same way, for the case of the $\mathrm{Fe}_{3} \mathrm{O}_{4}(111)$ layer, $\chi=29.50^{\circ}$ and $2 \theta=35.4^{\circ}$ to detect the (311) reflection. These azimuthal scans are collected as polar plots in Figures 3(b) and 3(d) to emphasize the symmetry of the hematite and magnetite layers on the $\mathrm{Au}(111)$ substrate.
The azimuthal scans of the hematite film in the $\alpha-\mathrm{Fe}_{2} \mathrm{O}_{3}(0001) /$ $\mathrm{Au}(111)$ layer and for the magnetite film in the $\mathrm{Fe}_{3} \mathrm{O}_{4}(111) /$ $\alpha-\mathrm{Fe}_{2} \mathrm{O}_{3}(0001) / \mathrm{Au}(111)$ bilayer show that both have the expected sixfold symmetry, revealing the epitaxial and singlecrystalline character of the films in their whole extent.

Our SRIM ${ }^{20}$ simulations show that the main damage is located at a depth of about $2-3 \mathrm{~nm}$. This is shallower than
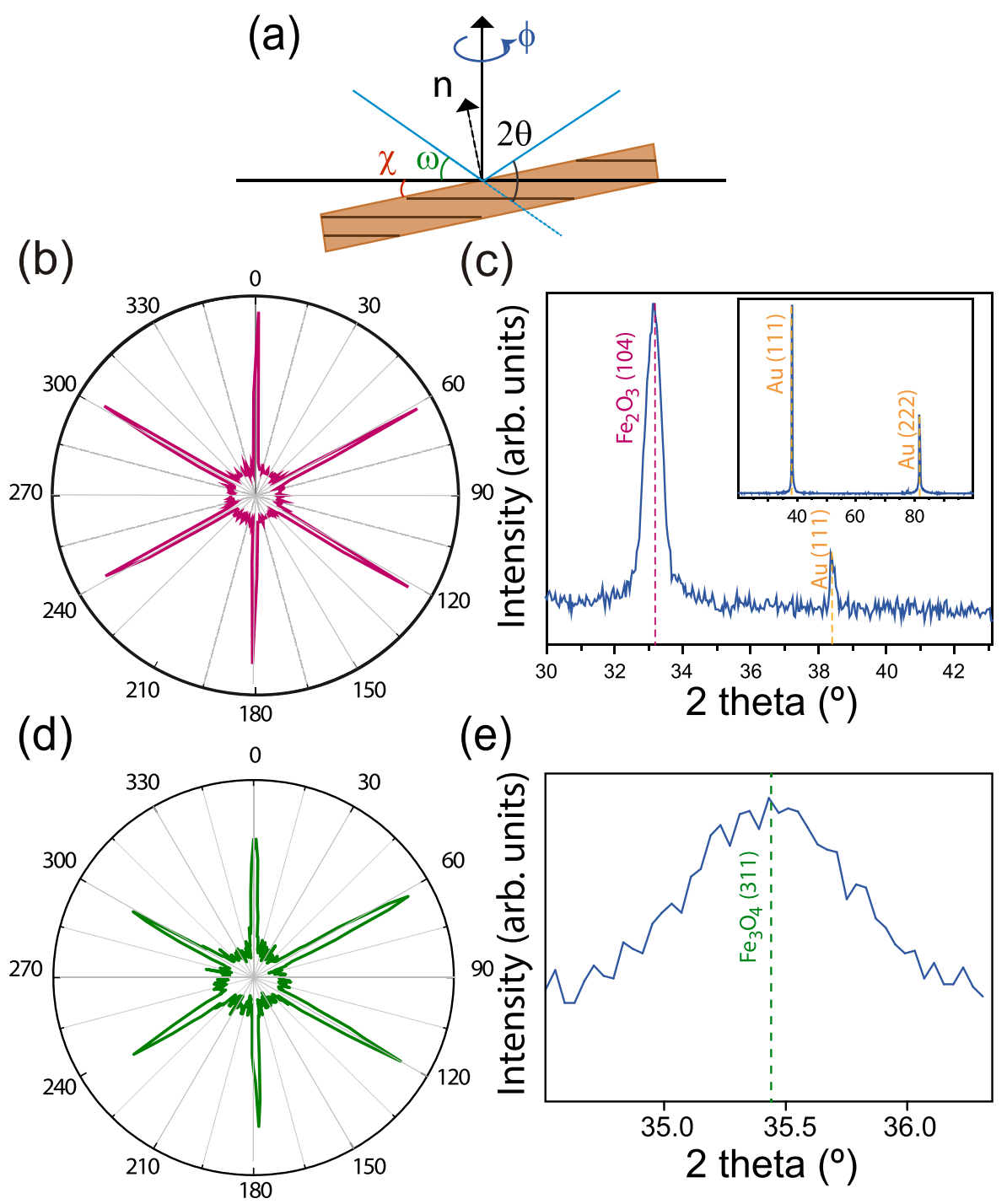

(e)

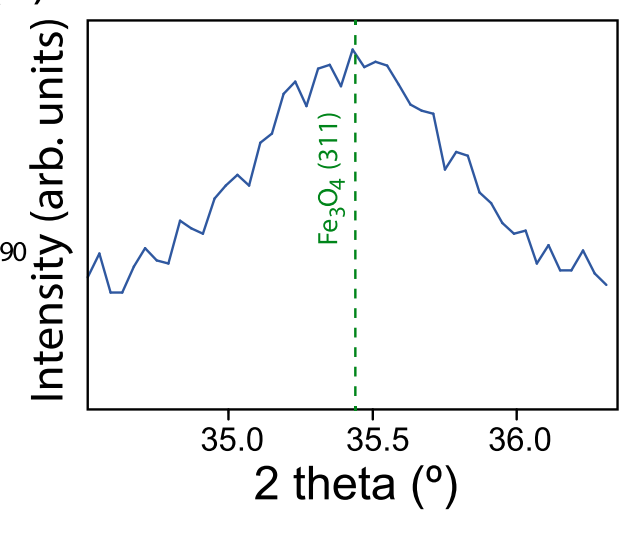

FIG. 3. (a) Scheme and angles used in the asymmetric XRD configuration for each phase. (b) and (d) Azimuthal XRD scans displayed as polar plots, probing the in-plane orientation of hematite (b) and magnetite (d) thin films on $\mathrm{Au}(111)$. A sixfold symmetry is present in both cases, showing the epitaxial and single-crystalline character of the whole films. In (c) and (e), only the reflections of the hematite and magnetite phases, respectively, are collected, although the system is indeed the magnetite/hematite bilayer. (c) The inset, conventional XRD scan of the sample, shows the very intense Au reflections. 
the whole magnetite thickness after its full transformation, but diffusion can be enhanced by ion irradiation. So, migration of defects at depths beyond the ion range must be responsible for the formation of a chemically reduced layer, with a thickness proportional to the ion dose. This can be justified primarily considering that every single colliding ion at $1 \mathrm{keV}$ produces a few point defects, ${ }^{21}$ mainly oxygen vacancies. The preferential sputtering of oxygen in iron oxides is a well reported phenomenon. ${ }^{22}$ Magnetite probably nucleates when the concentration of oxygen vacancies is too high to be accommodated in the hematite structure. But magnetite also undergoes oxygen loss once it is formed. Defect mobility must be present to compensate the oxygen loss of the upper layers $(2-3 \mathrm{~nm})$ of the magnetite film and to account for its growth. The species diffusing could be either $\mathrm{O}$ anions migrating from the $\mathrm{Fe}_{3} \mathrm{O}_{4} / \alpha-\mathrm{Fe}_{2} \mathrm{O}_{3}$ interface to the magnetite upper layers or $\mathrm{Fe}$ cations ${ }^{23}$ diffusing in opposite sense. Whatever is the existing mechanism, it effectively displaces the interface further into the bulk.

The epitaxial character of the bilayer relies on the oxygen planes. Both oxides crystallize in different structures (corundum for $\alpha-\mathrm{Fe}_{2} \mathrm{O}_{3}$ and inverse spinel for $\mathrm{Fe}_{3} \mathrm{O}_{4}$ ), and also the oxygen atoms are arranged in different sub-lattices (hcp and fcc, respectively). But, along the c direction $\left(\alpha-\mathrm{Fe}_{2} \mathrm{O}_{3}(0001)\right.$ and $\mathrm{Fe}_{3} \mathrm{O}_{4}(111)$ ), and although the stacking sequence of the oxygen planes along those directions differs, the close-packed oxygen planes are almost coincident. This reason, and the fact that the oxygen planes are almost coincident also to the $\mathrm{Au}(111)$ plane, eases the structural matching of both oxides.

We have performed magnetic measurements of the bilayer to reveal the magnetic character of the system grown.
In all measurements (MOKE, SQUID, and VSM), magnetization is measured with an in-plane applied magnetic field. The bilayer shows clear evidences of the Verwey transition, ${ }^{24}$ as shown in Figure 4(a), with hysteresis loops for different temperatures. At $300 \mathrm{~K}$ and $200 \mathrm{~K}$ (above the Verwey temperature $T_{V}$ ), the loops are similar, not displaying noticeable changes (except for some noise present in the regions approaching saturation). But at $T=100 \mathrm{~K}$, below $T_{V}$, the loop clearly becomes broader (from about $H_{c}=400 \mathrm{Oe}$ at RT to about $H_{c}=1000$ Oe at $100 \mathrm{~K}$ ). The increase in coercivity across the Verwey transition while cooling is well reported and due to changes in magnetostriction and magnetocrystalline anisotropy constants when the crystalline structure changes from a high- $\mathrm{T}$ cubic structure to the low- $\mathrm{T}$ monoclinic phase. Another consequence of the Verwey transition is shown in Figure 4(b) where both FC and ZFC curves display an increase in magnetization while crossing the transition around $T_{V}=130 \mathrm{~K}$. This value is slightly higher than the temperature reported for bulk single crystals $\left(T_{V}=122 \mathrm{~K}\right)$, which could be attributed to the interaction with the hematite substrate, as it has been reported for other substrates. ${ }^{25,26}$ The Verwey transition involves a structural distortion which can be strongly influenced by the stress present in thin films. The rise in the susceptibility across $T_{V}$ while heating is due to a sudden reduction of anisotropy. The clear transition in the loops at Figure 4(a) and the sharp increase in magnetization in ZFC/FC curves in Figure 4(b) suggest a good crystallinity of the magnetite films. Another expression of the continuous and epitaxial character of the films is that both FC and ZFC curves still lie far apart at room temperature since magnetite retains a high coercivity.

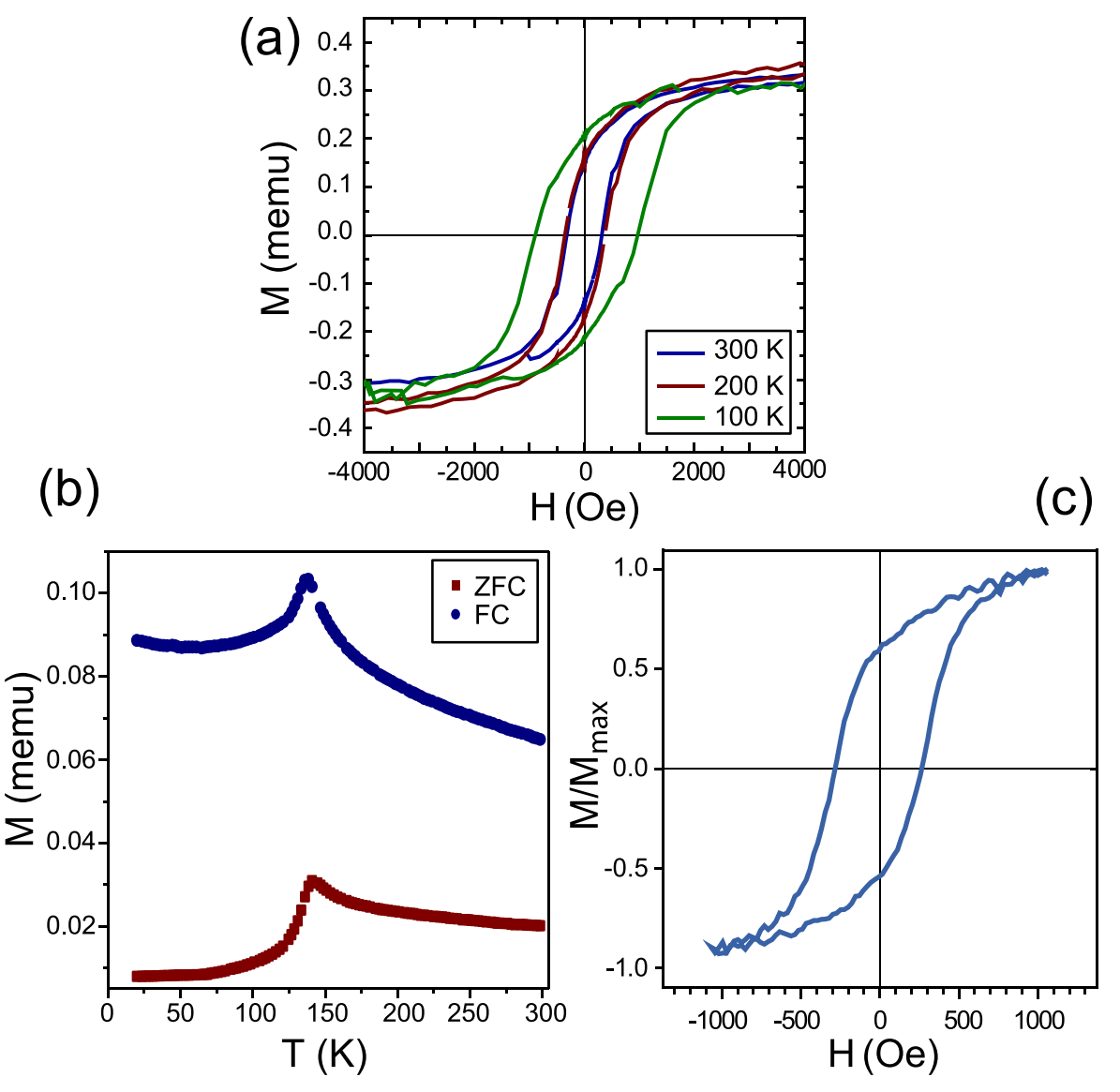

FIG. 4. Magnetic measurements performed on the magnetite/hematite bilayer. (a) Hysteresis loops of the sample performed with VSM at different temperatures, with an evident increase of coercivity below the Verwey transition of magnetite. (b) FC/ZFC curves measured with SQUID, showing the Verwey transition of the magnetite film. (c) Hysteresis loop measured with MOKE at RT. 
On the other hand, the absence of the Morin transition around $T_{M}=260 \mathrm{~K}$ can be ascribed to the higher saturation magnetization of magnetite (about a factor 200 compared to that of hematite), which may hinder the detection of magnetic signals stemming from hematite. Also, the Morin transition is truly absent in some cases, which has been attributed to strain effects ${ }^{27}$ or to a strong interaction between hematite and magnetite when the two oxides are present. ${ }^{10}$

To check that the measured magnetic signal originates at the surface of the sample (and thus at the bilayer), we have carried out MOKE measurements. Figure 4(c) shows the RT MOKE hysteresis loops of a bilayer and presents an information similar to that of Figure 4(a), although the origin of the information is more surface-sensitive in MOKE. It is evident that the hysteresis loops studied in these samples are mainly determined by the magnetite layer since hematite is a very weak ferromagnet (or canted antiferromagnet) at RT, above the Morin transition (the saturation magnetization of magnetite is around 200 times that of hematite). The coercive fields $H_{c}$ obtained with both VSM and MOKE (between 300 and 400 Oe at RT) are high compared to other magnetite layers studied in the literature with similar thicknesses, ${ }^{28-31}$ which could be partly due to coupling effects with the hematite substrate. Indeed, one of the most common manifestations of exchange effects between ferromagnetic (FM) and AFM layers is the enhancement of $H_{c}$, which is also observed in FiM/AFM structures. ${ }^{3,4}$

In summary, we have shown that low energy ion bombardment can be a convenient tool to fabricate an epitaxial bilayer of magnetite and hematite. Starting from an epitaxial hematite thin film, the process is driven by its progressive reduction during ion bombardment. This is induced by the higher sputtering yield of oxygen atoms and favoured by the good registry between the oxygen sub-lattices of both oxides. The process is reversible, and the magnetic response of the bilayer is mainly determined by the magnetite layer, which has a relatively high coercivity and shows a clear Verwey transition.

This work was supported by projects MAT2012-38045C04-03, FIS2014-61839-EXP, MAT2014-52477-C5-2-P and MAT2013-48009-C4-1-P from the Spanish Ministry of Economy and Competitiveness and a 2016 BBVA Foundation Grant for Researchers and Cultural Creators. S.R.-G. thanks a FPI MINECO fellowship. J. Romero from the C.A.I de Técnicas Físicas-UCM is acknowledged for the SQUID measurements.
${ }^{1}$ D. P. Norton, Mat. Sci. Eng. R. 43(5-6) 139-247 2004.

${ }^{2}$ H. Y. Hwang, Y. Iwasa, M. Kawasaki, B. Keimer, N. Nagaosa, and Y. Tokura, Nat. Mater. 11(2) 103-113 (2012).

${ }^{3}$ J. Nogués and I. K. Schuller, J. Magn. Magn. Mater. 192(2) 203-232 (1999).

${ }^{4} J$. Nogués, J. Sort, V. Langlais, V. Skumryev, S. Suriñach, J. S. Muñoz, and M. D. Baró, Phys. Rep. 422(3) 65-17 (2005).

${ }^{5}$ B. M. Pabón, J. I. Beltrán, G. Sánchez-Santolino, I. Palacio, J. LópezSánchez, J. Rubio-Zuazo, J. M. Rojo, P. Ferrer, A. Mascaraque, M. C. Muñoz, M. Varela, G. R. Castro, and O. Rodríguez de la Fuente, Nat. Comm. 6, 6147 (2015).

${ }^{6}$ X. H. Liu, W. B. Cui, W. Liu, X. G. Zhao, D. Li, and Z. D. Zhang, J. Alloys Compd. 475(1-2) 42-45 (2009).

${ }^{7}$ D. Tripathy, A. O. Adeyeye, and C. B. Boothroyd, J. Appl. Phys. 99, 08J105 (2006).

${ }^{8}$ W. B. Mi, E. Y. Jiang, and H. L. Bai, Appl. Phys. Lett. 93(13), 132504 (2008).

${ }^{9}$ P. Hajra, S. Basu, S. Dutta, P. Brahma, and D. Chakravorty, J. Magn. Magn. Mater. 321(14), 2269-2275 (2009).

${ }^{10}$ J. Ma, K. Chen, and X. Zhang, J. Appl. Phys. 111, 033925 (2012).

${ }^{11}$ C. Schlueter, M. Luebbe, A. M. Gigler, and W. Moritz, Surf. Sci. 605(23-24), 1986 (2011).

${ }^{12}$ Y. Tang, H. Qin, K. Wu, Q. Guo, and J. Guo, Surf. Sci. 609, 67-72 (2013).

${ }^{13}$ F. Genuzio, A. Sala, T. Schmidt, D. Menzel, and H.-J. Freund, Surf. Sci. 648, 177 (2016).

${ }^{14}$ M. Monti, B. Santos, A. Mascaraque, O. Rodríguez de la Fuente, M. A. Niño, T. O. Mentes, A. Locatelli, K. F. McCarty, J. F. Marco, and J. de la Figuera, J. Phys. Chem. C 116, 11539 (2012).

${ }^{15}$ D. L. A. de Faria, S. V. Silva, and M. T. De Oliveira, J. Raman Spectrosc. 28, 873 (1997).

${ }^{16}$ D. Bersani, P. P. Lottici, and A. Montenero, J. Raman Spectrosc. 30, 355 (1999).

${ }^{17}$ M. Lübbe, A. M. Gigler, R. W. Stark, and W. Moritz, Surf. Sci. 604, 679 (2010).

${ }^{18}$ A. Serrano Rubio, Modified Au-Based Nanomaterials Studied by Surface Plasmon Resonance Spectroscopy (Springer International Publishing, Switzerland, 2015).

${ }^{19}$ O. N. Shebanova and P. Lazor, J. Solid State Chem. 174, 424 (2003).

${ }^{20}$ J. F. Ziegler, M. D. Ziegler, and J. P. Biersack, Nucl. Instrum. Methods Phys. Res., Sect. B 268, 1818-1823 (2010).

${ }^{21}$ E. Carrasco, O. Rodríguez de la Fuente, M. A. González, and J. M. Rojo, Eur. Phys. J. B 40(4), 421-426 (2004).

${ }^{22}$ T. Choudhury, S. O. Saied, J. L. Sullivan, and A. M. Abbot, J. Phys. D: Appl. Phys. 22, 1185 (1989).

${ }^{23}$ G. S. Parkinson, Surf. Sci. Rep. 71, 272 (2016).

${ }^{24}$ F. Walz, J. Phys.: Cond. Matter 14(12) R285 (2002).

${ }^{25}$ S. Jain, A. O. Adeyeye, and C. B. Boothroyd, J. Appl. Phys. 97, 093713 (2005).

${ }^{26}$ S. Tiwari, R. Prakash, R. J. Choudhary, and D. M. Phase, J. Phys. D: Appl. Phys. 40, 4943 (2007).

${ }^{27}$ S. Gota, M. Gautier-Soyer, and M. Sacchi, Phys. Rev. B 64, 224407 (2001).

${ }^{28}$ Y. Zhou, C. McEvoy, R. Ramos, and I. V. Shvets, J. Appl. Phys. 99, 08J111 (2006).

${ }^{29}$ S. K. Arora, H. Wu, R. J. Choudhary, I. V. Shvets, O. N. Mryasov, H. Yao, and W. Y. Ching, Phys. Rev. B 77, 134443 (2008).

${ }^{30}$ S. K. Arora, H. Wu, H. Yao, W. Y. Ching, R. J. Choudhary, I. V. Shvets, and O. N. Mryasov, IEEE Trans. Magn. 44, 2628 (2008).

${ }^{31}$ Y. Zhai, L. Sun, Z. C. Huang, Y. X. Lu, G. D. Li, Q. Li, Y. B. Xu, J. Wu, and H. R. Zhai, J. Appl. Phys. 107, 09B110 (2010). 